\title{
Martin Kintzinger, Frank Rexroth, Jörg Rogge (dir.), Gewalt und Widerstand in der politischen Kultur des späten Mittelalters
}

\section{Gisela Naegle}

\section{(2) OpenEdition}

1 Journals

Édition électronique

URL : http://journals.openedition.org/ifha/8803

DOI : $10.4000 /$ ifha. 8803

ISSN : 2198-8943

\section{Éditeur}

IFRA - Institut franco-allemand (sciences historiques et sociales)

\section{Référence électronique}

Gisela Naegle, « Martin Kintzinger, Frank Rexroth, Jörg Rogge (dir.), Gewalt und Widerstand in der politischen Kultur des späten Mittelalters », Revue de l'IFHA [En ligne], Date de recension, mis en ligne le 02 novembre 2017, consulté le 24 septembre 2020. URL : http://journals.openedition.org/ifha/8803 DOI : https://doi.org/10.4000/ifha.8803

Ce document a été généré automatiquement le 24 septembre 2020.

(CIFHA 


\title{
Martin Kintzinger, Frank Rexroth, Jörg Rogge (dir.), Gewalt und Widerstand in der politischen Kultur des späten Mittelalters
}

\author{
Gisela Naegle
}

\section{RÉFÉRENCE}

Martin Kintzinger, Frank Rexroth, Jörg Rogge (dir.), Gewalt und Widerstand in der politischen Kultur des späten Mittelalters, Ostfildern: Jan Thorbecke Verlag (Konstanzer Arbeitskreis für mittelalterliche Geschichte, Vorträge und Forschungen, LXXX), 2015, 372 p., $56 €$ 
L'ouvrage collectif dirigé par Martin Kintzinger, Frank Rexroth et Jörg Rogge est issu d'un colloque du Konstanzer Arbeitskreis de 2009. Son sujet, la violence, a beaucoup retenu l'intérêt de l'historiographie récente. Le livre

Gewalt und Widerstand in der politischen Kultur des späten Mittelalters poursuit les réflexions que deux des éditeurs avaient déjà lancées avec «Gewalt gegen Könige. Macht und Mord im spätmittelalterlichen Europa» ( L La violence contre les rois. Pouvoir et assassinat en Europe à la fin du Moyen Âge ", 2004). Les douze articles sont encadrés par une introduction des trois éditeurs et des conclusions de Hermann Kamp. La définition retenue de la violence est étroite. Elle privilégie les formes et les pratiques de la violence corporelle et physique exercée par les personnes. Les éditeurs excluent la question de la violence structurelle engendrée par la configuration particulière d'une société. À l'échelle terminologique, ils discutent la différence entre potestas légitime, violentia et crudelitas, ainsi que les problèmes de traduction liés à ces notions. Par exemple, le mot allemand «Gewalt " peut exprimer aussi bien l'idée de «pouvoir légitime » que celle de « violence ».

Pour l'espace de la France, du Saint Empire et de l'Angleterre, sous le vocable du « rex crudelis ", Jean-Marie Moeglin étudie les formes du comportement violent des rois (du $\mathrm{XI}^{\mathrm{e}}$ au XIV $\mathrm{X}^{\mathrm{e}}$ siècle). Stefan Leder et Jenny Rahel Oesterle examinent l'espace de l'islam, l'importance $\mathrm{du}$ droit religieux, les conceptions d'honneur de sociétés tribales, et posent la question de savoir si ce système de droit basé sur le Coran, la scharia, et les doctrines savantes des érudits, théologiens et juristes permettent l'opposition et la révolte contre le gouvernant. Pour la Bohême, Uwe Tresp présente les tentatives de protéger les élections royales contre les ingérences violentes et les menaces. Il examine ainsi la situation qui a suivi la mort du dernier roi premyslide (1306-1307, l'assemblée électorale fut perturbée par des meurtres) et l'élection de Georges Podiebrad (1458). L'étude de Jörg Rogge se réfère au cas anglais et écossais. Au moins pour les chroniqueurs français du Moyen Âge, les "meurtriers des rois" anglais étaient qualifiés de cas à part. La présentation du sort des favoris (Günstlinge), qui est mentionné dans plusieurs contributions, est particulièrement digne d'intérêt. Ils étaient souvent de basse extraction. La noblesse, qui craignait pour sa propre influence et n'acceptait pas le pouvoir de ces parvenus, tenta de les éliminer. L'article de Karl Ubl porte sur la figure du tyran à l'époque du règne de Philippe le Bel (1285-1314). K. Ubl montre que ce roi sut intégrer et surmonter cette critique. En réagissant au moins partiellement aux reproches, à certains moments de son règne, il réussit même à les instrumentaliser pour stabiliser et renforcer son pouvoir. Le point de départ des réflexions de David Nirenberg sur le concept d'état d'exception (Ausnahmezustand) et sur le rôle des miracles est particulièrement remarquable. Confronté au massacre des Juifs à Valence en 1391, qui, du fait de leur statut juridique particulier, étaient sous sa 
protection spéciale, le roi Joan I $^{\text {er }}$ (qui régna sur la Catalogne, l'Aragon et Valence) préconisa d'abord une répression exemplaire et très violente. Par la suite, il y renonça et pardonna à la ville. Selon l'auteur, deux prétentions concurrentes de suspendre l'application de la loi se sont opposées: le pouvoir de la juridiction royale / la souveraineté royale et les prétentions de la ville, qui s'appuya sur l'allégation de miracles / l'intervention divine. Andreas Bihrer montre que, pour l'empire, l'instrumentalisation du meurtre du roi Albert $\mathrm{I}^{\mathrm{er}}$ (1308) a eu des répercussions politiques plus importantes que l'acte en soi. Dans ce cas, l'historiographie médiévale postérieure livra différentes interprétations et adapta ses récits aux intérêts politiques du moment.

L'article très riche de Franck Collard sur la sémantique de l'empoisonnement va dans le même sens. Il est difficile de donner des chiffres exacts sur ce type de meurtre, mais il fut très important dans le discours politique. Cette arme occulte des faibles et des femmes heurta les valeurs du temps. Très apprécié des princes et même des rois pour éliminer leurs adversaires, l'empoisonnement ne fut pas accepté comme un moyen légitime et honorable $\mathrm{du}$ combat contre un prince ou un roi tyrannique. L'hypothèse selon laquelle le royaume français, en tant que système "fermé ", où le mode de la succession royale ne permettait pas d'issues "variables", encouragea davantage les tentatives d'empoisonnement du roi, mérite d'être approfondie.

Par leur thématique très intéressante, mais moins liée à l'exercice concret de la violence corporelle et physique, qui est au centre des autres contributions, les articles de Thorsten Hiltmann (qui présente une synthèse et des exemples issus de ses recherches sur les " autres rois », dont les rois de ribauds, roi de la Basoche, roi des sots, roi des ménestrels, roi des merciers, etc.) et de William Caferro (rituels militaires, dialogue sur l'honneur, mises en scène et images déshonorantes dans le contexte des guerres interurbaines de l'Italie communale) forment une catégorie un peu à part. Ce volet thématique élargit le champ d'investigation à la sphère des répercussions et des éventuelles conséquences violentes des actes de l'exercice du pouvoir au sens large. L'article de Nicolas Offenstadt sur les crieurs montre que leurs publications pouvaient déclencher des actes violents. Même si, contrairement aux rois cruels, aux justiciers, aux empoisonneurs et autres, ils n'étaient pas des " acteurs » directs de la violence, ils pouvaient être eux-mêmes attaqués à cause du contenu des messages publiés.

Dans l'ensemble, il s'agit d'un volume fort intéressant qui fournit un grand nombre de pistes de recherches et invite à la poursuite de cette réflexion.

INDEX

Thèmes : Histoire des États et des pouvoirs, Histoire de la culture, Historiographie/

Méthodologie, Histoire des mentalités

Index chronologique : Moyen Âge 
AUTEURS

GISELA NAEGLE

Gießen / Paris 